\title{
Correlation between tumor regression grade and rectal volume in neoadjuvant concurrent chemoradiotherapy for rectal cancer
}

\author{
Hong Seok Lee, MD, Doo Ho Choi, MD, Hee Chul Park, MD, Won Park, MD, Jeong II Yu, MD, \\ Kwangzoo Chung, PhD \\ Department of Radiation Oncology, Samsung Medical Center, Sungkyunkwan University School of Medicine, Seoul, Korea
}

Purpose: To determine whether large rectal volume on planning computed tomography (CT) results in lower tumor regression grade (TRG) after neoadjuvant concurrent chemoradiotherapy (CCRT) in rectal cancer patients.

Materials and Methods: We reviewed medical records of 113 patients treated with surgery following neoadjuvant CCRT for rectal cancer between January and December 2012. Rectal volume was contoured on axial images in which gross tumor volume was included. Average axial rectal area (ARA) was defined as rectal volume divided by longitudinal tumor length. The impact of rectal volume and ARA on TRG was assessed.

Results: Average rectal volume and ARA were $11.3 \mathrm{~mL}$ and $2.9 \mathrm{~cm}^{2}$. After completion of neoadjuvant CCRT in 113 patients, pathologic results revealed total regression (TRG 4) in 28 patients (25\%), good regression (TRG 3) in 25 patients (22\%), moderate regression (TRG 2) in 34 patients (30\%), minor regression (TRG 1) in 24 patients (21\%), and no regression (TRG0) in 2 patients (2\%). No difference of rectal volume and ARA was found between each TRG groups. Linear correlation existed between rectal volume and TRG ( $p=0.036)$ but not between ARA and TRG ( $p=0.058)$.

Conclusion: Rectal volume on planning CT has no significance on TRG in patients receiving neoadjuvant CCRT for rectal cancer. These results indicate that maintaining minimal rectal volume before each treatment may not be necessary.

Keywords: Rectal cancer, Neoadjuvant chemoradiotherapy, Rectal volume, Tumor regression grade

\section{Introduction}

The incidence of colorectal cancer has continued to increase in South Korea. Between 1999 and 2010, the annual percentage change in the age-standardized incidence rate was 5.9\% in both sexes. Colorectal cancer has become the second most common cancer among men and third most common cancer among women [1]. Local recurrence is a major factor that influences prognosis of rectal cancer patients, occurring in 10\% $-15 \%$ of patients according to recent studies [2]. Preoperative concurrent chemoradiotherapy (CCRT) demonstrated higher local control and reduced toxicity compared to postoperative CCRT in recent studies [3]. Currently, preoperative CCRT is considered the treatment of choice in locally advanced (T34 or node positive) rectal cancer. Downstaging following neoadjuvant radiation shows a favorable survival outcome

Received 1 April 2016, Revised 31 May 2016, Accepted 17 June 2016.

Correspondence: Doo Ho Choi, MD, Department of Radiation Oncology, Samsung Medical Center, Sungkyunkwan University School of Medicine, 81 Irwon-ro, Gangnam-gu, Seoul 06351, Korea. Tel: +82-2-3140-2612, Fax: +82-2-3140-2619, E-mail: doho.choi@samsung.com

(C) This is an Open Access article distributed under the terms of the Creative Commons Attribution Non-Commercial License (http://creativecommons.org/ licenses/by-nc/4.0/) which permits unrestricted non-commercial use, distribution, and reproduction in any medium, provided the original work is properly cited.

www.e-roj.org 
$[4,5]$. Also, tumor regression grade (TRG) is considered as an independent prognostic factor for rectal cancer [6]. Fokas et al. [7] suggested that higher TRG correlates with better diseasefree survival and lower distant metastasis.

Higher radiation dose is responsible for increase in rectal tumor response and improved sphincter preservation [8]. However, in clinical situation, various factors negatively affect the conformality of radiation delivery. Teh et al. [9] reported that the radiation dose at the air-tissue interface of the rectum, dilated with a rectal balloon, is lower in comparison to the same phantom without an air cavity. When we prepare for delivery of neoadjuvant CCRT in rectal cancer patients, some of the planning computed tomographies (CTs) have large rectal air volume inside the target volumes. If we suppose that large air volume in the rectum persists while delivering radiotherapy $(\mathrm{RT})$, we can presume that the target volume of the rectal wall may be under-dosed, thus affecting the response rate of the tumor.

Our hypothesis in the present study was as follows. A large rectal volume on planning $\mathrm{CT}$ for RT may lead to insufficient dose coverage at the rectal wall region, thus negatively affecting the TRG of rectal cancer. This study was intended to confirm the necessity of routine rectal volume evaluation and emptying during neoadjuvant CCRT of rectal cancer patients.

\section{Materials and Methods}

\section{Patient population}

From January to December 2012, among 138 patients who were treated with surgery following neoadjuvant CCRT for rectal cancer at Samsung Medical Center, 113 patients were included in this study. Patients with previous chemotherapy history or previous radiation history, patients who did not complete chemoradiation, and patients whose radiation record not available were excluded from the analysis. The clinical stage of rectal cancer was evaluated by rectum magnetic resonance imaging (MRI), based on the American Joint Committee on Cancer criteria (6th edition). Patients with distant metastasis were excluded from the study.

\section{Treatment characteristics}

All patients underwent simulation and treatment in the prone position and were scanned with CT for RT planning. All patients received $44 \mathrm{~Gy}$ to primary tumor and pelvic nodes in fractional dose of $2 \mathrm{~Gy}$. In cases which surgical resection for lateral pelvic node was not considered feasible, an additional boost dose of 10-16 Gy with reduced field was delivered for
25 patients (22\%). Three-dimensional conformal RT plans routinely consisted of two lateral beams with 10-MV photons and one posterior beam with 4-MV photon.

Chemotherapy was delivered in two modalities. Fifty-four patients (48\%) were continuously injected with 5-fluorouracil (5-FU) $\left(400 \mathrm{mg} / \mathrm{m}^{2} /\right.$ day) and leucovorin $\left(20 \mathrm{mg} / \mathrm{m}^{2} /\right.$ day $)$ during the first week and last week of radiotherapy. One cycle of chemotherapy consisted of 3 days of treatment in a neoadjuvant setting. An alternative regimen of capecitabine ( $850 \mathrm{mg} / \mathrm{m}^{2} /$ twice a day) for 35 days during radiotherapy was prescribed for the other 59 patients (52\%).

Following neoadjuvant CCRT with an interval of 2 to 3 months, 97 (86\%) had low anterior resection, $6(5 \%)$ had abdominal perineal resection, 7 (7\%) had intersphincteric resection, and $3(3 \%)$ had local excision.

\section{Tumor regression grade}

The pathologic response after CCRT was determined by one pathologist for all patients. Pathologic grading of primary tumor regression was performed by determining the amount of fibrotic tissue compared to viable tumor, separated into five groups of TRG, according to Dworak et al. [10]. The five groups of TRG were as follows: grade 4, total regression (no viable tumor cells; only fibrotic mass); grade 3, good regression (dominant fibrosis outgrowing tumor mass, $>50 \%$ tumor regression); grade 2, moderate regression (dominant tumor mass with obvious fibrosis in 26\%-50\% of tumor mass); grade 1, minor regression (dominant tumor mass with obvious fibrosis $\leq 25 \%$ of tumor mass); grade 0 , no regression (fibrosis completely absent).

Tumor downstaging was also evaluated, comparing pretreatment clinical stage and pathologic stage.

\section{Assessment of rectal volume}

Rectal volume was calculated as summation of all gas volume in axial images containing gross tumor volume (GTV), and was automatically contoured on the axial images of planning CT using the auto-contouring function of the pinnacle planning system (Pinnacle, ver. 9.10; Philips Medical Systems, Andover, MA, USA). Fig. 1 shows two examples of planning CT with distended rectum, contoured with GTV, clinical target volume (CTV) and rectal volume.

Average axial rectal area (ARA) was calculated as rectal volume divided by longitudinal tumor length. Longitudinal tumor length was defined as perpendicular distance between upper and lower boundaries of GTV in axial CT images.

The trend of rectal volume change throughout the 

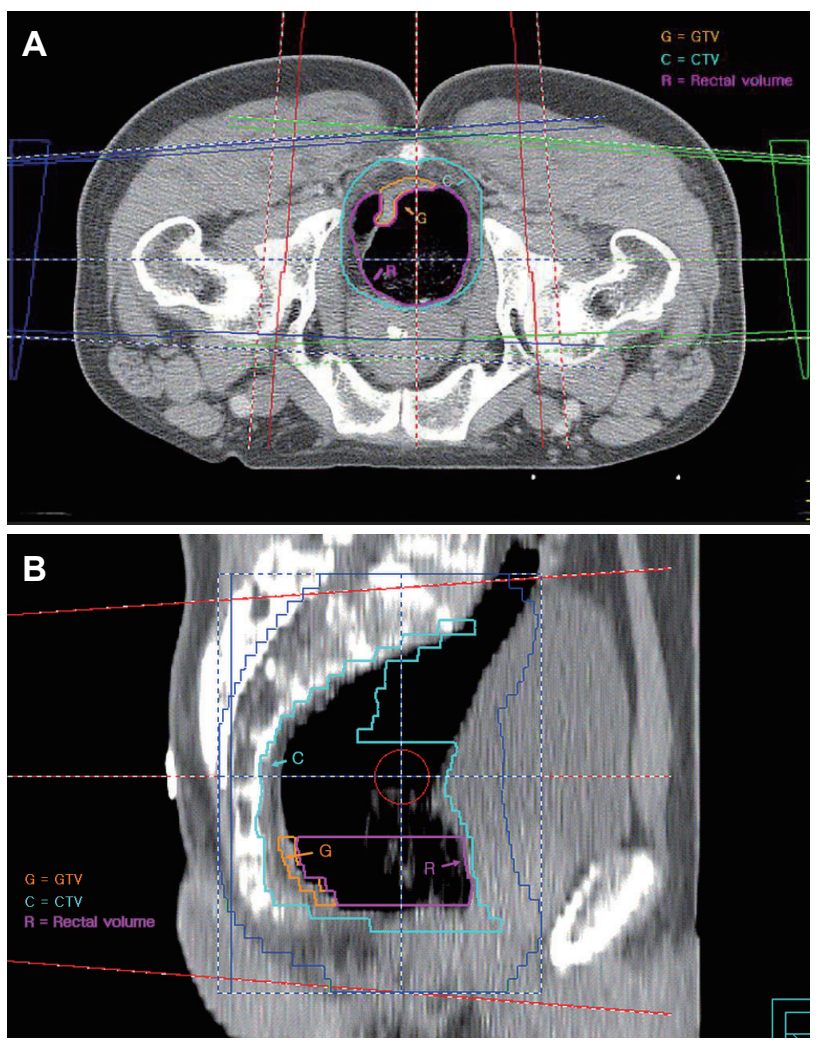

Fig. 1. An example of axial view (A) and sagittal view (B) of planning computed tomography images showing large rectal volume. Each image was contoured with gross tumor volume (orange), clinical target volume (turquoise), and rectal volume (purple). Rectal volume was defined as summation of all gas volume in axial images containing gross tumor volume. GTV, gross tumor volume; CTV, clinical target volume.

Table 1. Patient characteristics

\begin{tabular}{lc}
\multicolumn{1}{c}{ Characteristic } & No. (\%) \\
\hline Age (yr), median (range) & $59(30-81)$ \\
Sex & \\
Male & $77(68.1)$ \\
Female & $36(31.9)$ \\
Distance from the anal verge (cm) & \\
$<5$ & $65(57.5)$ \\
$\geq 5$ & $48(42.5)$ \\
Clinical tumor staging ${ }^{a)}$ & \\
I & $5(4.4)$ \\
II & $5(4.4)$ \\
IIIA & $8(7.1)$ \\
IIIB & $38(33.6)$ \\
IIIC & $57(50.4)$ \\
Total dose (Gy) & $88(72.7)$ \\
44 & $25(22.1)$ \\
$54-60$ &
\end{tabular}

${ }^{a)}$ American Joint Committee on Cancer 6th edition. treatment was assessed for 29 patients whose mid-treatment planning CT was obtainable. Mid-treatment CT images were acquired after receiving 18-22 fraction of radiation, with identical treatment set-up to the primary CT images. Rectal volume evaluation of mid-treatment $\mathrm{CT}$ images was performed with the same technique used for primary CT images. We compared two CT images of each patient to evaluate the consistency of rectal volume.

\section{Statistical analysis}

Paired t-test and Pearson correlation were used to confirm the consistency of rectal volume. Kruskal-Wallis test and student t-test were used to compare rectal volume between different TRG groups. The linear relationship between rectal volume and pathologic response (including TRG and downstaging) was analyzed using Spearman correlation. Clinicopathologic factors in each TRG group was compared with Pearson chi-square test and Kruskal-Wallis test.

All tests were two-sided and considered statistically significant for $p$-value less than 0.05. We used IBM SPSS ver. 20.0 (IBM, Armonk, NY, USA) for the analysis.

\section{Results}

\section{Patient characteristics}

The patient group had a total of 113 people: 77 male (68\%) and 36 female (32\%). The median age of the population was 59 years (range, 30 to 81 years). Clinical stage before CCRT was stage I in 5 patients (4\%), stage II in 5 patients (4\%), and stage III in 103 patients (91\%) (Table 1).

\section{Consistency of rectal volume}

Among 113 patients, we were able to assess mid-treatment planning CT for 29 patients (24\%). The average value and 95\% confidence interval (Cl) of rectal volume was $14.7 \mathrm{~mL}(95 \%$ $\mathrm{Cl}, 9.01$ to $20.4 \mathrm{~mL})$ for the primary images and $10.1 \mathrm{~mL}(95 \%$ $\mathrm{Cl}, 4.61$ to $15.6 \mathrm{~mL}$ ) for the mid-treatment images. Paired t-test showed no difference of rectal volume between the two groups $(p=0.147)$. Also, a linear correlation was found between rectal volumes before and after the treatment (Pearson correlation coefficient $=0.43, p=0.022$ ), suggesting that the tendency of rectal volume would persist throughout the course of RT.

\section{Tumor response and rectal volume}

Pre-treatment clinical stage and pathologic stage was compared for 113 patients. In this study, 99 patients (88\%) 
showed downstaging and 14 patients (12\%) showed no stage reduction. Three cases (3\%) showed progression: stage I to II in one case and stage II to IIIA in two cases (Table 2).

After the completion of neoadjuvant CCRT, pathologic results showed total regression (TRG 4) in 28 patients (25\%), good regression (TRG 3) in 25 patients (22\%), moderate regression (TRG 2) in 34 patients (30\%), minor regression (TRG 1) in 24 patients (21\%), and no regression (TRG 0) in 2 patients (2\%).

Average rectal volume and ARA measured on planning CT were $11.1 \mathrm{~mL}$ (range, 0 to $65.0 \mathrm{~mL}$ ) and $2.9 \mathrm{~cm}^{2}$ (range, 0 to $19.8 \mathrm{~cm}^{2}$ ), respectively. Proximal tumors (anal verge $\geq 5 \mathrm{~cm}$ ) had larger rectal volume than distal tumors (anal verge $<5$ $\mathrm{cm}$ ) (mean value: distal $8.6 \mathrm{~mL}$, proximal $14.5 \mathrm{~mL} ; \mathrm{p}=0.034$ ) However, no correlation existed between rectal volume and pre-treatment stage $(p=0.155)$ or GTV volume $(p=0.161)$.

Average rectal volumes of each TRG group were $9.0 \mathrm{~mL}$ (TRG 4), $8.8 \mathrm{~mL}$ (TRG 3), $13.7 \mathrm{~mL}$ (TRG 2), $11.7 \mathrm{~mL}$ (TRG 1), and 19.2 $\mathrm{mL}$ (TRG 0). A borderline difference of rectal volume was found between 5 TRG groups ( $p=0.091$ by Kruskal-Wallis test). No

Table 2. Comparison between pre-treatment stage and postoperative pathologic stage

\begin{tabular}{|c|c|c|c|c|c|c|c|}
\hline \multirow{2}{*}{ Clinical stage $\mathrm{e}^{\mathrm{a})}$} & \multicolumn{7}{|c|}{ Postoperative pathologic stage $\mathrm{e}^{\mathrm{a})}$} \\
\hline & yp0 & ypl & ypll & yplllA & ypllIB & ypllIC & Total \\
\hline 1 & 4 & - & $1^{\text {b) }}$ & - & - & - & 5 \\
\hline ॥ & 3 & - & - & $2^{b)}$ & - & - & 5 \\
\hline IIIA & 4 & 3 & - & 1 & - & - & 8 \\
\hline IIIB & 9 & 13 & 7 & 3 & 6 & - & 38 \\
\hline IIIC & 12 & 19 & 12 & 3 & 7 & 4 & 57 \\
\hline Total & 32 & 35 & 20 & 9 & 13 & 4 & 113 \\
\hline
\end{tabular}

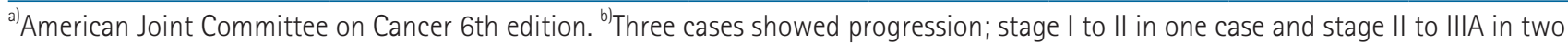
cases.

Table 3. Correlation between TRG and clinicopathologic factors

\begin{tabular}{|c|c|c|c|c|c|c|}
\hline Characteristic & $\begin{array}{l}\text { TRGO } \\
(n=2)\end{array}$ & $\begin{array}{c}\text { TRG1 } \\
(n=24)\end{array}$ & $\begin{array}{c}\text { TRG2 } \\
(n=34)\end{array}$ & $\begin{array}{c}\text { TRG3 } \\
(n=25)\end{array}$ & $\begin{array}{c}\text { TRG4 } \\
(n=28)\end{array}$ & p-value \\
\hline Age (yr), median & 58.5 & 53 & 59.5 & 60 & 59.5 & 0.406 \\
\hline Sex & & & & & & 0.434 \\
\hline Male & 2 & 18 & 25 & 14 & 18 & \\
\hline Female & 0 & 6 & 9 & 11 & 10 & \\
\hline Tumor site & & & & & & 0.467 \\
\hline$>$ Anal verge $5 \mathrm{~cm}$ & 2 & 7 & 14 & 12 & 11 & \\
\hline$\leq$ Anal verge $5 \mathrm{~cm}$ & 0 & 17 & 20 & 13 & 17 & \\
\hline Pre-CCRT CEA (ng/mL) & 3.3 & 4.5 & 3.2 & 5.7 & 3.8 & 0.435 \\
\hline Pre-CCRT stage & & & & & & 0.015 \\
\hline । & 0 & 0 & 1 & 0 & 4 & \\
\hline ॥ & 0 & 0 & 2 & 0 & 3 & \\
\hline IIIA & 0 & 2 & 2 & 3 & 1 & \\
\hline IIIB & 0 & 8 & 13 & 10 & 7 & \\
\hline IIIC & 2 & 14 & 16 & 12 & 13 & \\
\hline CCRT-to-surgery interval (mo), average & 128 & 101 & 98 & 89 & 92 & 0.239 \\
\hline Lymphovascular invasion & 1 & 10 & 5 & 4 & 0 & 0.002 \\
\hline Perineural invasion & 0 & 11 & 3 & & 0 & $<0.001$ \\
\hline Total dose (Gy) & & & & & & 0.982 \\
\hline 44 & 2 & 18 & 27 & 19 & 22 & \\
\hline $54-60$ & 0 & 6 & 7 & 6 & 6 & \\
\hline Rectal volume $(\mathrm{mL})$, average & 19.2 & 11.7 & 13.7 & 8.8 & 9.0 & 0.091 \\
\hline ARA $\left(\mathrm{cm}^{2}\right)$, average & 2.9 & 2.7 & 3.9 & 2.4 & 2.3 & 0.126 \\
\hline
\end{tabular}

TRG, tumor regression grade; CCRT, concurrent chemoradiotherapy; CEA, carcinoembryonic antigen; ARA, average axial rectal area. 
significant difference between TRG groups was found for ARA ( $p=0.126$ by Kruskal-Wallis test) (Table 3 ).

A linear correlation was found between TRG and rectal volume (Spearman correlation efficient $=-0.20, p=0.036$ ), but not between downstaging and rectal volume $(p=0.231)$. Only marginal relationship was found between TRG and ARA (Spearman correlation efficient $=-0.18, p=0.058$ ).

To confirm the clinical impact of large rectal volume, we divided the patients into two groups at the median value of rectal volume (large volume group, $>4.3 \mathrm{~mL}$; small volume group, $\leq 4.3 \mathrm{~mL}$ ). The large volume group had smaller TRG value $(2.31 ; 95 \% \mathrm{Cl}, 2.02$ to 2.60$)$ compared to small volume group $(2.62 ; 95 \% \mathrm{Cl}, 2.33$ to 2.91$)$. However, the difference was not statistically significant ( $p=0.145$ by t-test). We used the Receiver Operating Characteristic curve analysis to determine the optimal cut-off point for estimating TRG value. As a result, rectal volume of $0.15 \mathrm{~mL}$ had the highest sum of sensitivity (0.817) and specificity (0.491). We selected this value as a second cutoff point for further analysis. The group containing larger rectal gas within the rectum (rectal volume $\geq 0.15 \mathrm{~mL}$, $\mathrm{n}=37)$ had smaller TRG value $(2.25 ; 95 \% \mathrm{Cl}, 2.00$ to 2.50$)$ compared to the group that had almost none of the gas $(2.92$; $95 \% \mathrm{Cl}, 2.57$ to 3.27), with statistical significance ( $p=0.003$ by t-test). Also, when comparing complete response (TRG 4) with other TRG groups (TRG 0-3), the group containing no rectal gas (rectal volume $<0.15 \mathrm{~mL}$ ) also showed more complete response rate with statistical significance ( $p=0.040$ by t-test).

Subgroup analysis regarding clinical stage and distance from anal verge yielded no significant findings.

\section{Group comparison based on TRG}

Based on TRG, clinicopathologic factors of patients were analyzed (Table 3$)$. Pre-CCRT stage ( $p=0.015)$, Iymphovascular invasion ( $p=0.002)$, and perineural invasion $(p<0.001)$ were significantly correlated with TRG. However, distance from anal verge, radiation to surgery interval, and total irradiated dose had no correlation with TRG.

\section{Discussion and Conclusion}

The benefits of preoperative radiotherapy, especially 5-FUbased chemoradiation, have been clearly established in patients with locally advanced rectal cancer $[11,12]$. Tumor response rate and pathologic downstaging are prognostic factors of rectal cancer, affecting local tumor control and survival rates $[13,14]$. For rectal cancer, significant doseresponse relationship is demonstrated in radiation dose up to $70 \mathrm{~Gy}$, showing that adequate dose delivery is critical for tumor control [15]. Thus, approaches to achieve sufficient dose coverage to the tumor are meaningful, such as in the current study, in which we focused on the impact of large rectal gas volume on tumor control. It have been reported that rectal distention has a significant impact on treatment failure in prostate cancer [16]. Furthermore, many efforts have been made to reduce the variations of rectal volume in prostate cancer, such as laxatives, daily direct gas removal, or diet protocols [17-19]. Target dislocation is the main issue in the case of prostate cancer; nevertheless, we can presume that large rectal air volume may increase the variation in rectal dose-volume parameters in rectal cancer cases as well.

Dose perturbation near the air-tissue surfaces is one of the main difficulties in delivering RT precisely. Perturbation of the electron fluence occurs in this region of electronic disequilibrium [20]. This concern has been emphasized in head-and-neck tumors due to presence of an air cavity $[21,22]$. Dose perturbation near the air cavity is strongly dependent on X-ray energy, field size, depth, and size of cavity. For example, the Monte Carlo calculations show dose reductions of $40 \%$ and $19 \%$ at 0.05 and $2 \mathrm{~mm}$, respectively, beyond the air-water interface for a 3-cm diameter cylindrical air tube irradiated by a single $5 \mathrm{~cm} \times 5 \mathrm{~cm} 15-\mathrm{MV}$ beam. These reductions became roughly half for parallel-opposed beam arrangement, being 21\% and 11\% for the same depths [20]. However, in an in vivo setting, there are some factors that decrease this dose perturbation. Intra-fractional variation in the internal geometry can result in the statistical averaging of dose distribution. Also, the posterior beam used in this study, which is perpendicular to lateral beams, will decrease the dose reduction effect near the air-tissue surface. The wedges used in the lateral beams may also contribute to diminishing the effects of dose reduction.

In this study, we measured the rectal volume of axial images containing GTV, to investigate the effect of dose perturbation to tumor control in clinical setting. We confirmed that rectal gas volume does not change significantly over fractions of radiation treatment, by reviewing mid-treatment planning CT of 29 patients.

From the analysis of 113 patients who received neoadjuvant CCRT for rectal cancer, we found out that rectal volume in planning CT was not correlated with tumor regression grade. Patients with large rectal volume resulted in lower average TRG value, but there was no statistical significance. We did found a difference of TRG between groups divided at cut-off value of $0.15 \mathrm{~mL}$ in rectal volume in this study. However, we are 
reluctant to correlate this finding towards clinical significance, because the cut-off value is too close to zero, which makes it problematic to have statistical significance. Also, we expected that the dose reduction effect will have clinical impact at larger rectal volumes, but the small value of $0.15 \mathrm{~mL}$ does not fit in with the radiophysics basis assumed in this study.

Accordingly, our result does not support that reducing rectal volume before each treatment fraction would be beneficial for patients with large rectal volume. The dose reduction effect from air cavity may not be significant enough to affect the clinical outcomes of the patients. Also, there was no difference of TRG between distant and proximal tumors. Rectal movement in rectal cancer patients mainly occurs in upper rectum, especially in anterior and lateral directions [23]. Although proximal tumors had larger rectal volumes in our study, this anatomic variation throughout the treatment seems to contribute in diminishing the dose reduction effect.

While undergoing neoadjuvant CCRT, total irradiated dose was between $44 \mathrm{~Gy}$ to $60 \mathrm{~Gy}$. All boost radiation for 25 patients who received more than $44 \mathrm{~Gy}$ was targeting at lateral pelvic nodes, and was planned to minimize additional dose to the rectum. Thus, the only dose scheme irradiated to the main mass was $44 \mathrm{~Gy}$, making it difficult to analyze the dose-response relationship in this patient group.

The main limitation of this study is that inter-fractional images were not acquirable in majority of the patients. We made effort to show the consistency of rectal volume by analyzing the mid-treatment rectal volume in 29 patients, but its statistical power would not be strong enough to be applied for the population group. Ki et al. [17] obtained daily megavoltage CT images in 40 prostate cancer patients while receiving radical $R T$, and demonstrated that there was no significant variation of rectal volume during the course of RT. Likewise, if there were data of periodic CT images during treatment, we could assess the consistency of rectal volume more precisely. Furthermore, patient group with persistent large rectal volume can be selected to analyze the correlation with tumor response. Therefore, we suggest further studies with sufficient and periodic image data to confirm our results.

\section{Conflict of Interest}

No potential conflict of interest relevant to this article was reported.

\section{References}

1. Jung KW, Won YJ, Kong HJ, Oh CM, Seo HG, Lee JS. Cancer statistics in Korea: incidence, mortality, survival and prevalence in 2010. Cancer Res Treat 2013;45:1-14.

2. Klint $A$, Engholm $G$, Storm $H H$, et al. Trends in survival of patients diagnosed with cancer of the digestive organs in the Nordic countries 1964-2003 followed up to the end of 2006. Acta Oncol 2010;49:578-607.

3. Sauer R, Becker $H_{1}$ Hohenberger $W$, et al. Preoperative versus postoperative chemoradiotherapy for rectal cancer. N Engl J Med 2004;351:1731-40.

4. Janjan NA, Abbruzzese J, Pazdur R, et al. Prognostic implications of response to preoperative infusional chemoradiation in locally advanced rectal cancer. Radiother Oncol 1999;51:153-60.

5. Berger $C$, de Muret A, Garaud $P$, et al. Preoperative radiotherapy (RT) for rectal cancer: predictive factors of tumor downstaging and residual tumor cell density (RTCD): prognostic implications. Int J Radiat Oncol Biol Phys 1997;37:619-27.

6. Dhadda AS, Dickinson P, Zaitoun AM, Gandhi N, Bessell EM. Prognostic importance of Mandard tumour regression grade following pre-operative chemo/radiotherapy for locally advanced rectal cancer. Eur J Cancer 2011;47:1138-45.

7. Fokas $E$, Liersch $T$, Fietkau $R$, et al. Tumor regression grading after preoperative chemoradiotherapy for locally advanced rectal carcinoma revisited: updated results of the CAO/ARO/ Al0-94 trial. J Clin Oncol 2014;32:1554-62.

8. Gerard JP, Chapet 0 , Nemoz C, et al. Improved sphincter preservation in low rectal cancer with high-dose preoperative radiotherapy: the lyon R96-02 randomized trial. J Clin Oncol 2004;22:2404-9.

9. Teh BS, Dong L, McGary JE, Mai WY, Grant W 3rd, Butler EB. Rectal wall sparing by dosimetric effect of rectal balloon used during intensity-modulated radiation therapy (IMRT) for prostate cancer. Med Dosim 2005;30:25-30.

10. Dworak O, Keilholz L, Hoffmann A. Pathological features of rectal cancer after preoperative radiochemotherapy. Int J Colorectal Dis 1997;12:19-23.

11. Sauer R, Liersch T, Merkel S, et al. Preoperative versus postoperative chemoradiotherapy for locally advanced rectal cancer: results of the German CAO/ARO/AIO-94 randomized phase III trial after a median follow-up of 11 years. J Clin Oncol 2012;30:1926-33.

12. Folkesson J, Birgisson H, Pahlman L, Cedermark B, Glimelius B, Gunnarsson U. Swedish Rectal Cancer Trial: long lasting benefits from radiotherapy on survival and local recurrence rate. J Clin Oncol 2005;23:5644-50.

13. Moore HG, Gittleman AE, Minsky BD, et al. Rate of pathologic 
complete response with increased interval between preoperative combined modality therapy and rectal cancer resection. Dis Colon Rectum 2004;47:279-86.

14. Chan AK, Wong A, Jenken D, Heine J, Buie D, Johnson D. Posttreatment TNM staging is a prognostic indicator of survival and recurrence in tethered or fixed rectal carcinoma after preoperative chemotherapy and radiotherapy. Int J Radiat Oncol Biol Phys 2005;61:665-77.

15. Appelt AL, Ploen J, Vogelius IR, Bentzen SM, Jakobsen A. Radiation dose-response model for locally advanced rectal cancer after preoperative chemoradiation therapy. Int J Radiat Oncol Biol Phys 2013;85:74-80.

16. de Crevoisier $R$, Tucker $S L$, Dong $L$, et al. Increased risk of biochemical and local failure in patients with distended rectum on the planning $\mathrm{CT}$ for prostate cancer radiotherapy. Int J Radiat Oncol Biol Phys 2005;62:965-73.

17. Ki Y, Kim WT, Nam J, et al. Probiotics for rectal volume variation during radiation therapy for prostate cancer. Int J Radiat Oncol Biol Phys 2013;87:646-50.

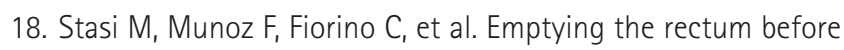
treatment delivery limits the variations of rectal dose-volume parameters during 3DCRT of prostate cancer. Radiother Oncol 2006;80:363-70.

19. Smitsmans MH, Pos FJ, de Bois J, et al. The influence of a dietary protocol on cone beam CT-guided radiotherapy for prostate cancer patients. Int J Radiat Oncol Biol Phys 2008;71:1279-86.

20. Li XA, Yu C, Holmes T. A systematic evaluation of air cavity dose perturbation in megavoltage $x$-ray beams. Med Phys 2000;27:1011-7.

21. Ostwald PM, Kron T, Hamilton CS. Assessment of mucosal underdosing in larynx irradiation. Int J Radiat Oncol Biol Phys 1996:36:181-7.

22. Niroomand-Rad A, Harter KW, Thobejane S, Bertrand K. Air cavity effects on the radiation dose to the larynx using Co-60, $6 \mathrm{MV}$, and $10 \mathrm{MV}$ photon beams. Int J Radiat Oncol Biol Phys 1994;29:1139-46.

23. Chong I, Hawkins M, Hansen V, et al. Quantification of organ motion during chemoradiotherapy of rectal cancer using cone-beam computed tomography. Int J Radiat Oncol Biol Phys $2011 ; 81: e 431-8$. 